REVESCO. Revista de Estudios Cooperativos

ISSN: $1885-8031$

\title{
Emprendimiento social en jóvenes: análisis de su relación con otras formas de participación social ${ }^{1}$
}

\author{
María Celeste Dávila ${ }^{2}$, Anna Zlobina ${ }^{3}$ y Amparo Serrano Pascual ${ }^{4}(0)$
}

Recibido: 15 de diciembre de 2020 / Aceptado: 2 de febrero de 2021 / Publicado: 29 de abril de 2021

Resumen. El emprendimiento social es un fenómeno relativamente novedoso en nuestro país, pero a pesar de su importancia para el tercer sector y de las potenciales conexiones con otras formas más tradicionales de participación social, apenas se encuentran estudios que hayan abordado el análisis de tales relaciones. El propósito de este trabajo es analizar la disposición de los jóvenes a desarrollar emprendimiento social, estudiar la vinculación que tiene el emprendimiento con otras formas de participación social, y la relación que mantiene con la obligación moral de participar socialmente para determinar hasta qué punto el paradigma del emprendimiento también ha calado entre los jóvenes como una fórmula para afrontar los problemas sociales. A través de un cuestionario en papel, 261 estudiantes universitarios aportaron información sobre las cuestiones previamente apuntadas. Los resultados hallados evidencian la relación entre emprendimiento social y otras formas de participación social. La intención de emprender socialmente es inferior a la intención de poner en práctica otras formas de participación fundamentalmente cívicas. Finalmente, la obligación moral sólo predice la intención de emprender a través de su relación con la intención de desarrollar otras formas de participación social. Se discuten los resultados hallados.

Palabras clave: Voluntariado; Activismo; Asociacionismo; Participación política; Desempleo juvenil.

Claves Econlit: C83; D64; E24; J62; J64; J31.

\section{[en] Social entrepreneurship in young people: analysis of their relationship with other forms of social participation}

\begin{abstract}
Social entrepreneurship is a relatively novel phenomenon in our country, but despite its importance to the third sector and potential connections to other more traditional forms of social participation, there are hardly any studies that have addressed the analysis of such relationships. The purpose of this paper is to analyze the disposition of young people to engage in social entrepreneurship, to study the relationships between entrepreneurship and other forms of social participation, and its relationship with the moral obligation to participate socially in order to determine the extent to which the entrepreneurship paradigm has penetrated among young people as a formula for addressing social problems. Through a paper questionnaire, 261 university students provided information on the previous issues. The results evidence the existence of significant relationships between social entrepreneurship and other forms of social participation. The intention to start social entrepreneurship is lower to the intention to implement other forms of fundamentally civic participation. Finally, moral obligation only predicts the intention to undertake socially through its relationship with the intention of developing other forms of social participation. The results are discussed.
\end{abstract}

Keywords: Volunteerism; Activism; Voluntary association membership; Political participation; Youth unemployment.

Sumario. 1. Introducción. 2. Metodología. 3. Resultados. 4. Discusión. 5. Conclusiones. 6. Referencias bibliográficas.

Cómo citar. Dávila, M.C.; Zlobina, A.; Serrano Pascual, A. (2021) Emprendimiento social en jóvenes: análisis de su relación con otras formas de participación social. REVESCO. Revista de Estudios Cooperativos, vol. $138, \quad$ e75562. https://dx.doi.org/10.5209/reve.75562.

1 Esta investigación forma parte del proyecto El emprendimiento como estrategia de lucha contra el desempleo juvenil: de la política institucional a la vivencia y experiencia de los sujetos financiado por el Ministerio de Economía, Industria y Competitividad. Plan Nacional. Programa Excelencia (2018). Referencia: CSO2017-82839-P.

2 Universidad Complutense de Madrid, España.

Dirección de correo electrónico: mcdavila@ucm.es

3 Universidad Complutense de Madrid, España.

Dirección de correo electrónico: azlobina@cps.ucm.es.

4 Universidad Complutense de Madrid, España.

Dirección de correo electrónico: aserranop@cps.ucm.es 


\section{Introducción}

El desempleo juvenil es un problema social que se ha exacerbado con la crisis económica y que se ha visto acrecentado también tras la pandemia del COVID-19. Según datos de Eurofound (2020), los jóvenes son y serán los más afectados por esta situación. Durante las últimas décadas el trabajo ha sufrido un proceso de redefinición como categoría central del ordenamiento social, lo que conlleva que este problema social pueda tener importantes implicaciones a largo plazo en aspectos tales como la salud y el bienestar, la vinculación social y el estatus económico, por ejemplo (Eurofound, 2020).

En los últimos años, el paradigma del emprendimiento se ha presentado como una solución al desempleo en general y al desempleo juvenil, en particular. Este paradigma es incorporado, en gran medida, en las recientes orientaciones de las políticas de lucha contra el desempleo entre los jóvenes, y más específicamente, en la Estrategia de Emprendimiento y Empleo joven (EEEJ) y en el programa de Garantía Juvenil implementados por parte del Gobierno de España. Estos programas constituyen una importante expresión del creciente énfasis político en promover un cambio en la mentalidad de los jóvenes e inducir actitudes emprendedoras como condición de lucha contra el desempleo (Cabasés, Pardell y Feixa, 2018; Escudero, 2016; Henar, 2018; Martínez, 2017; Rodríguez y Verd, 2018; Rodriguez, Serrano y Martínez, 2020; Romero y Milone, 2016; Santamaría y Carbajo, 2019; Santos, 2014; Serrano y Fernández, 2018; Suárez, 2017; Úbeda y Sánchez, 2018).

Estos discursos institucionales orientados al emprendimiento enaltecen la independencia y pueden funcionar como interpelaciones morales (ser activo, dueño de la propia vida, etc.). La promoción de la autonomía e independencia se convierten en este sentido en un eje clave a la hora de plantear soluciones frente al desempleo juvenil (Santos, 2014; Santamaría y Carbajo, 2019; Serrano y Martín-Martín, 2017). Estos mandatos emprendedores van más allá de una mera propuesta de nuevos modos de trabajar y de salida de la crisis del mercado de trabajo. Suponen también procesos de subjetivación, es decir, un proyecto de transformación de la mentalidad de los jóvenes.

Por tanto, el paradigma del emprendimiento se está orientando no solo a combatir el desempleo juvenil sino también a transformar las culturas laborales, y a inducir nuevos arquetipos de sujeto. Gran parte de estas políticas dirigidas a promover el emprendimiento se han traducido en programas de ingeniería actitudinal: inclusión de la formación emprendedora en el sistema educativo en sus diversas fases (primaria, secundaria y universitaria); promoción de modelos de rol en los medios y en la industria cultural; extensión de la figura del emprendedor en el seno del marketing de los influencer o de la industria cultural terapéutica (Rodríguez y Borges, 2018). El principio que comparten estos diversos programas es la necesidad de promover sujetos activos, que sean capaces de tomar las riendas de sus vidas a la hora de afrontar problemas, y promotores de dinamismo e innovación. Su objetivo es fomentar una cultura emprendedora entre los jóvenes (Eurofound, 2015).

Esta orientación que convierte a la crisis del empleo en una crisis cultural ha sido objeto de un intenso debate en el seno de la literatura científica. Algunos autores consideran este énfasis en promover el emprendimiento entre los jóvenes como la solución más viable ante la extensión del problema del desempleo juvenil y destacan sus bondades (Alvord, Brown y Letts, 2004; Díaz y Lejarriaga, 2018; Makua, CuencaAmigo y San Salvador, 2017; Sánchez, Martín, Bel y Lejarriaga, 2018; Velasco, Estrada, Pabón y Tójar, 2019; por ejemplo). Sin embargo, otras investigaciones adoptan una perspectiva crítica, por entender que esta interpelación cultural participa en un proyecto de racionalidad neoliberal que promueve la individualización del trabajo, la psicologización de la exclusión social y el reforzamiento de la vulnerabilización política del sujeto (por ejemplo, Briales, 2017; Martínez, 2017; Santamaría y Carbajo, 2019; Santos, 2014; Serrano y Martín, 2017; Suárez, 2017; Úbeda y Sánchez, 2018; Valenzuela-García, Molina, Lubbers, Escribano y Fuentes, 2019).

En este trabajo no se pretende profundizar en este debate, sino abordar una dimensión vinculada con la extensión de dicha cultura que se denomina emprendimiento social, y que en cierta forma podría eludir estos riesgos a los que hemos aludido. Además de las racionalidades que inspiran el énfasis en la promoción del emprendimiento, se ha destacado la capacidad de dicho paradigma para desbordar sus fronteras semánticas vinculadas con una dimensión puramente económica y extender su racionalidad a otros espacios sociales, como son la educación, el gobierno social, los movimientos sociales y ciudadanos, etc. pudiendo inducir la colonización de los modos de pensar y gobernar los problemas sociales.

Teniendo en cuenta la dimensión metanarrativa de la noción de emprendimiento, esto es, su capacidad para extenderse a otros ámbitos que el meramente económico, nos interesa analizar la disposición de los jóvenes a proyectarse hacia el emprendimiento social. Concretamente, los objetivos del trabajo son analizar la disposición de los jóvenes universitarios a desarrollar emprendimiento social, analizar la vinculación que tiene con otras formas de participación social, y, finalmente, la relación que mantiene con objetivos que trascienden la mera inserción laboral, como por ejemplo, la obligación moral de participación e implicación social, con el propósito de determinar hasta qué punto y de qué manera el referencial emprendedor está calando también en la forma de afrontar los problemas sociales. 
Para ello, en primer lugar, se plantearán algunas de las conclusiones a las que ha llegado la literatura acerca de la extensión y significado del emprendimiento social. Aunque las delimitaciones semánticas de dicha noción resultan difusas, parece haber un cierto consenso en que el discurso del emprendimiento ha tenido un impacto en los modos de entender la participación social. Seguidamente, se aborda el papel del emprendimiento social como una alternativa al empleo y se analizará las potenciales vinculaciones del emprendimiento social con otras formas de participación social. Finalmente, se presenta una breve revisión sobre los principales hallazgos relativos a los fundamentos morales que parecen guiar al emprendimiento social. Esta revisión previa plantea diversas cuestiones que son analizadas en la segunda parte de este artículo, donde se describe el diseño metodológico y se presentan y discuten los resultados hallados en la investigación desarrollada para dar respuesta a las cuestiones inicialmente planteadas.

\subsection{Emprendimiento social: un área de estudio en creciente expansión}

La revisión del estudio sobre emprendimiento social revela que se trata de un área de trabajo en expansión, pero que se encuentra muy fragmentada y carece de un marco teórico coherente (Gawell, 2013; Gupta, Chauhanb, Paulc, y Jaiswald, 2020; Hota, Subramanian y Gopalakrishnan, 2020; Pareja, 2013; Santos, 2012; Seelos y Mair, 2004; Weerawardena y Mort, 2006). Sumado a ello se ha intentado conceptualizar en muy diferentes contextos (sector público, empresa, organizaciones de acción social, etc.) (Hota et al., 2020; Kraus, Filser, O'Dwyer y Shaw, 2014; Wang y Aaltio, 2017; Weerawardena y Mort, 2006), y la aproximación a su conceptualización difiere mucho dependiendo de la perspectiva de análisis. Puede suponer diferentes cosas para diferentes personas (Guzman y Trujillo, 2008; Pareja, 2013). Por ejemplo, Alvord et al. (2004) identifican tres aproximaciones en su conceptualización.

Desde el mundo de la empresa se concibe el emprendimiento social como una aplicación del emprendimiento empresarial a objetivos sociales. Los emprendedores sociales se esfuerzan por crear valor social a través de modelos de negocio empresariales (Hota et al, 2020; Seelos y Mair, 2004). Definiciones que encajarían en esta perspectiva serían, por ejemplo, la aportada por McMillan (2003), que describe el emprendimiento social como un proceso mediante el cual la creación de una nueva empresa de negocios conduce a la mejora de la riqueza social para que tanto la sociedad como el emprendedor se beneficien, o la aportada por Seelos y Mair (2005), quienes consideran que "el emprendimiento social combina el ingenio del espíritu empresarial tradicional con la misión de cambiar la sociedad" (p.241).

Una segunda acepción de este concepto entiende al emprendimiento social como innovación para el cambio social (Alvord et al., 2004). Desde este planteamiento, Mair y Noboa (2003, 2006) describen que el emprendimiento social implica aproximaciones innovadoras para abordar problemas relativos a la educación, el medio ambiente, el comercio justo y los derechos humanos. Austin, Stevenson y Wei-Skillern (2006) lo definen como una actividad innovadora, que crea valor social. En esta misma dirección, la conceptualización que maneja la Fundación Ashoka encajaría perfectamente, ya que concibe a los emprendedores sociales como líderes de la innovación social.

Finalmente, la tercera aproximación a su conceptualización se vincula con las anteriores y concibe el emprendimiento como un catalizador de la transformación social. Alvord et al. (2004) describen que el emprendimiento social puede producir pequeños cambios a corto plazo que den lugar a grandes cambios a largo plazo a través de la interdependencia que existe entre los diferentes elementos del sistema social. Una intervención en un elemento crítico de ese sistema puede convertirse en una especie de "palanca" que genere una transformación social de mayor magnitud. Conseguir una transformación social sostenible necesariamente lleva a considerar el aspecto innovativo de la idea y una movilización de recursos propia de la gestión empresarial.

A pesar de la ambigüedad y polisemia que caracterizan al concepto, se pueden identificar ciertos elementos clave que comparten todas las definiciones: su objetivo es crear valor social y la actividad se caracteriza por la innovación o la creación de algo nuevo. Sumado a ello, el motor central del emprendimiento social es el problema concreto que se trata de abordar, pero el canal o vehículo particular a través del cual se aborda el problema puede ser muy diferente. El vehículo no tiene por qué tener una forma jurídica particular; la forma organizativa que toma una empresa social estará fundamentada en el formato que sea capaz de movilizar más eficazmente los recursos para abordar el problema. De esta forma, hay una importante heterogeneidad de tipos de actividad que pueden ser etiquetadas como emprendimiento social y se pueden encontrar ejemplos de emprendimiento social en sectores sin ánimo de lucro, de negocios o gubernamentales (Austin et al., 2006).

Además de analizar la polisemia que caracteriza al emprendimiento social, los temas en los que se ha centrado su estudio han sido muy diversos (definiciones y aproximaciones conceptuales, dimensiones del emprendimiento social, misiones $\mathrm{u}$ objetivos principales, modelos del emprendimiento social, responsabilidad social corporativa, educación, características de los emprendedores sociales, procesos y recursos utilizados, impacto, etc.). A pesar de ello, Gupta et al. (2020) identifican que en los últimos años se ha incrementado sustancialmente el interés por el estudio del impacto social del emprendimiento social, la 
innovación, las estrategias y modelos de negocio, y la creación y difusión de valor. Respecto a las metodologías utilizadas en la investigación, la aproximación cualitativa, especialmente los estudios de casos, es la que parece dominar, lo que demuestra la necesidad de estudios cuantitativos complementarios (Fernández, López y San Martín, 2020; Gupta et al., 2020; Hota et al., 2020). Sumado a ello, Kraus et al. (2014) describen que la mayoría de los artículos publicados antes de 2014 eran fundamentalmente conceptuales, y que los estudios empíricos eran escasos.

En España, aunque la economía social y solidaria ya poseía un largo recorrido, el emprendimiento social, como tal, es un sector relativamente novedoso (Valenzuela-García et al., 2019). Bosma, Schøtt, Terjesen y Kew (2016) muestran que España es uno de los países europeos con menores niveles de implicación en actividades de emprendimiento social. La prevalencia de la actividad empresarial social naciente es España según estos autores está por debajo del $2 \%$ en la población adulta.

\subsection{El emprendimiento social como alternativa al empleo}

La promoción del emprendimiento ha sido una de las orientaciones políticas más relevantes en el momento actual para hacer frente al desempleo juvenil, colectivo particularmente afectado por la destrucción de puestos de trabajo tras la crisis económica. Promovido por las instituciones europeas, a partir del 2012 se ponen en marcha una serie de iniciativas dirigidas a apoyar la inserción laboral de este colectivo. La transposición de estas iniciativas europeas en el caso español ha dado lugar a la Estrategia de Emprendimiento y Empleo Joven (EEEJ) (Real Decreto-Ley 4/2013) cuyo elemento central es el sistema de Garantía Juvenil. El fomento de la cultura emprendedora constituye uno de los principales ejes de este programa, que se ve apoyado financieramente por la Iniciativa de Empleo Juvenil y por el Fondo Social Europeo.

Se trata de iniciativas dirigidas, entre otros objetivos, a promover e incentivar el emprendimiento como un modo alternativo de inserción en el mercado de trabajo por parte de los jóvenes. Estas medidas comprenden diversos ejes de actuación (exenciones y bonificaciones para la promoción del autoempleo, e impulsar un cambio de mentalidad y orientación laboral, Rodríguez y Ramos, 2016; Rodríguez y Verd, 2018), adoptando como una línea prioritaria la promoción de la cultura emprendedora. En este contexto de representación del desempleo juvenil, se ha considerado al emprendimiento social como un modo de emprender que permite conjugar dos objetivos sociales: la creación de valor social y favorecer la empleabilidad de los jóvenes (Díaz y Lejarriaga, 2018).

Tan, Williams y Tan (2005) plantean un continuo con relación al emprendimiento social, donde en un extremo estaría la búsqueda únicamente del beneficio de la sociedad frente a, en el otro extremo, un emprendimiento social donde se busca tanto el beneficio personal como el social. La combinación entre creación de valor social, por un lado, y la creación de valor comercial o de mercado, por otro, crea una tensión entre las metas sociales y financieras, y esto ha llevado a muchos investigadores a analizar el emprendimiento social desde un planteamiento híbrido (Elsayed, 2018). Precisamente, las experiencias de emprendimiento social entre los jóvenes que pretenden promover su empleabilidad tendrían ese carácter híbrido.

El emprendimiento social no sólo se ha presentado como una alternativa al empleo para los jóvenes (ver por ejemplo, National Agency for European Educational Programmes and Mobility, 2017), sino también para otros colectivos que puedan estar expuestos a un mayor riesgo de exclusión social como son las personas con diversidad funcional (Harris, Renko y Caldwell, 2014; Ortiz y Olaz, 2019) o las mujeres (Haugh y Talwar, 2016; Kapusuz, Çavu y Pekkan, 2018), por ejemplo. En este sentido, se asume el emprendimiento social como una fórmula de empleo o autoempleo que contribuye a cambiar las estructuras de vulnerabilización en las que estos grupos están insertos, ayudándoles a superar barreras para conseguir la inclusión social. Pero se conoce muy poco sobre cómo el impacto del emprendimiento social a nivel individual se transforma en cambio social. Algunos trabajos han descrito que el empoderamiento tiene un papel mediador en esa relación (Haugh y Talwar, 2016), aunque este planteamiento también ha tenido importantes detractores (Boeri, 2018).

Pero ¿Cómo ven los jóvenes universitarios esta alternativa al empleo? ¿Cuál es su disposición a emprender socialmente? Como se ha descrito previamente, uno de los objetivos de este trabajo es analizar el grado de intención de los jóvenes de realizar emprendimiento social en un futuro próximo.

\subsection{Relación con otras formas de participación social}

El planteamiento inicial que se ha presentado con relación al emprendimiento social aporta ciertas claves que aluden a una conexión con otras formas de participación social que pueden contribuir igualmente a crear valor, afrontar los problemas sociales y transformar la sociedad. Un ejemplo de ello son las "Robin Hood 
Stores"5, donde el emprendimiento se funde con el activismo ("Robin Hood Stores: cuando hacer la compra es activismo anticapitalista”, 2020).

En relación a las conexiones entre emprendimiento social y otras formas de participación social, se han planteado fundamentalmente tres ejes de reflexión.

En primer lugar, el emprendimiento individual, es decir, el desarrollado de forma individual sin la colaboración de otras personas, domina las narrativas sobre emprendimiento social, pero tal y como se ha argumentado en numerosos estudios, mucho del emprendimiento es colaborativo o colectivo (por ejemplo, Montgomery, Dancin y Dancin, 2012; ver en Pless, 2012). La noción de acción colectiva para promover el cambio social no es nueva; un ejemplo de ello son los estudios sobre movimientos sociales. De hecho, Mair y Marti (2006) describen que ciertos insights de la literatura sobre movimientos sociales han sido aplicados al estudio del emprendimiento social. Tanto el emprendimiento como los movimientos sociales están orientados a la transformación social. En esta línea, Montesano (2016) analiza tres iniciativas de transformación social que combinan características de movimiento social y de empresas sociales. Siguiendo la aportación inicial de Mair y Marti (2006), este autor amplía las definiciones existentes sobre emprendimiento social, añadiendo el elemento de "movimientos sociales".

En segundo lugar, el emprendimiento social ha sido asociado con un amplio rango de procesos de cambio social (Haugh y Talwar, 2016), y quizás precisamente la relación con esos procesos es lo que puede conectar o vincular el emprendimiento social con otras formas de participación social como puede ser el activismo o el voluntariado. Makua et al. (2017) analizan la asociación entre prácticas de ocio solidario (voluntariado, por ejemplo) y emprendimiento social. Hockerts (2017) describe que la experiencia previa con problemas sociales a través del trabajo en organizaciones sociales es un predictor de la intención de emprender socialmente. Carrasco y Buendía (2020) muestran la fuerte relación existente entre el sector cooperativo, como fórmula de emprendimiento social, y la prevalencia de activismo político en un país. En la misma línea, los trabajos de Alkon (2018), Hildebrant (2011), Lee (2018), y Ngouyamsa (2016) (ver en Carrasco y Buendía, 2020) muestran dicha relación entre activismo y algunas formas de emprendimiento social. Martin y Osberg (2007) describen que el emprendimiento social y el activismo tienen un mismo motor: su movilización a partir de un problema social, y probablemente un emprendedor social y un activista compartan muchas características. Sin embargo, se diferencian en la naturaleza de la orientación de la acción. En el caso del activismo, el objetivo es, en mayor medida, ejercer influencia en otros (gobiernos, consumidores, etc.) para que sean esos otros los que lleven a cabo las acciones necesarias. Es decir, se promueve el cambio a través de acciones indirectas. En cambio, en el emprendimiento social se ponen en práctica medidas directas.

Finalmente, en tercer lugar, muchas entidades sociales u ONGs, que son el marco de actuación de diversas formas de participación social (voluntariado, por ejemplo), han incorporado el emprendimiento social en sus funciones, en muchos casos, como una fórmula para aumentar sus fuentes de financiación (Andersson y Self, 2015; Austin et al., 2006; Elsayed, 2018; Ko y Liu, 2020; Mair y Noboa, 2003; Seelos y Mair, 2004; Weerawardena y Mort, 2006). Stecker (2014) describe que la aplicación de los principios de emprendimiento social y de empresa social puede mejorar la sostenibilidad de las ONGs, al tiempo que mejora su capacidad de gestión y misión.

Estos tres elementos contribuyen a que los límites entre lo que se considera emprendimiento social y otras formas de participación social queden diluidos o desdibujados, y se genere una cierta confusión a la hora de etiquetar cada tipo de experiencia. En cambio, algunos autores plantean que las diferencias entre diversas experiencias y formas de participación social se centran en algo más esencial, que va más allá de ciertas divergencias o conexiones concretas. Elsayed (2018) describe que el emprendimiento social ha proliferado como una alternativa a las formas tradicionales de implicación cívica. De hecho, en su trabajo analiza la relación entre movimientos sociales y emprendimiento social no como dos fenómenos separados, sino como diferentes formas de abordar el mismo objetivo: promover una transformación social. En esta línea, Dees (2012) plantea incluso que el emprendimiento social supone una nueva cultura a la hora de abordar los problemas sociales en contraposición a la cultura de la caridad. En la cultura de la caridad, una acción desinteresada se desarrolla con el objetivo de beneficiar a otra persona por la que se siente compasión y esto lleva al que ayuda a sentirse satisfecho. En cambio, en la cultura del emprendimiento se busca la utilidad de los resultados y la excelencia de los métodos empleados para solucionar el problema social. Sin embargo, según este autor, el éxito del emprendimiento social dependerá de la medida en que estas dos culturas, y sus correspondientes sistemas de valores, consigan alinearse de tal forma que la satisfacción personal por dar o ayudar pueda contribuir de forma inteligente a resolver un problema social.

Se trata de una cadena de supermercados en Alemania montada por dos jóvenes que muestra un modelo de negocio donde los clientes pagan con su membresía o con horas de trabajo en los locales, contribuyendo a generar de esa forma un sentido de comunidad. Al mismo tiempo, luchan contra el cambio climático y la pobreza extrema, haciendo donaciones sobre los beneficios obtenidos a organizaciones que combaten estas problemáticas, y promueven igualmente la sostenibilidad vendiendo productos ecológicos. 
A pesar de las conexiones entre el emprendimiento social y otras fórmulas de transformación social, ni los análisis bibliométricos de Kraus et al. (2014) y de Hota et al. (2020), ni la revisión de Gupta et al. (2020) sobre el estudio del emprendimiento social identifican esta relación como un área de interés, ni señalan algún estudio cuyo objetivo sea explícitamente analizarla.

La presente investigación plantea, como segundo objetivo, analizar la relación que tiene el emprendimiento social con otras formas de participación social. Concretamente, propone estudiar la relación entre la intención de emprender socialmente y la intención de desarrollar otras formas de participación social en el futuro, y en qué medida el desarrollo previo de ciertas conductas de participación social se asocia con la disposición a emprender socialmente.

\subsection{Obligación moral}

Kraus et al. (2014) describieron que el análisis de las características de los emprendedores sociales ha sido una de las principales áreas de trabajo con relación al estudio del emprendimiento social. Diversos autores consideran que estas características son diferentes a las de otro tipo de emprendedores (Mair y Naboa, 2003; Portuguez, Valenzuela y Navarro, 2018; por ejemplo). Concretamente, Mair y Naboa (2003) describen que los emprendedores sociales están motivados por un fuerte deseo de cambiar la sociedad, y se caracterizan por el inconformismo con el estatus quo, el altruismo, y por la necesidad de responder a sus propios principios y ser responsables socialmente.

En el emprendimiento social están muy presentes valores morales, sociales y medioambientales; con frecuencia este emprendimiento se basa en motivos éticos y de responsabilidad moral (Mair y Marti, 2006; Valenzuela-García et al., 2019). Por ejemplo, Mair y Noboa $(2003,2006)$ señalan al juicio u obligación moral como uno de los elementos clave que impulsa la intención de emprender socialmente, y lo definen como un proceso cognitivo que motiva a una persona a ayudar a otras en la búsqueda de un bien común. Hockerts (2017) comprueba, en una muestra de estudiantes universitarios, que hay una relación significativa entre obligación moral e intención de emprender socialmente. Mair y Marti (2009) estudiaron a emprendedores sociales que trabajaban en condiciones de vacío institucional en países en desarrollo y argumentaron que la obligación moral hacia los necesitados era una dimensión clave que impulsaba su labor en este tipo de entornos. Nga y Shamuganathan (2010) identificaron que, entre las dimensiones que pueden caracterizar a los emprendedores sociales, está la "visión social", que hace referencia al sentido de obligación moral de los emprendedores, que les lleva a ver la oportunidad de intervención. Por último, Smith, Kistruck y Cannatelli (2016) utilizan el concepto de intensidad moral, es decir, en qué medida se experimenta un imperativo moral para tomar la decisión de ampliar una iniciativa de emprendimiento social.

La obligación moral se ha definido como una decisión personal de participar en una acción específica basada en un deber ser, esto es, en la creencia de que ello es lo que se debe hacer. De esta forma, sería la motivación que se experimenta para poner en práctica un comportamiento que se considera correcto (Sabucedo, Dono, Alzate y Seoane, 2018). Sabucedo et al. (2018) muestran que la obligación moral es el predictor más efectivo de la intención y la participación política real, y como se ha descrito previamente, también parece tener un papel importante en la predicción del emprendimiento social.

La obligación moral se cristaliza tanto en las disposiciones internas de los individuos (empatía, valores, etc.) como en sus experiencias sociales (Zlobina y Sánchez, 2020). Mair y Noboa $(2003,2006)$ señalan que factores como la exposición a experiencias sociales que confrontan a las personas con las necesidades, valores y puntos de vista de otros; la magnitud percibida de las consecuencias (daño o bien realizado a un individuo, por ejemplo); y el consenso social (nivel de acuerdo sobre la bondad o maldad de un acto concreto) afectan al nivel de obligación moral. En esta línea, el consenso social o las normas sociales tienen un papel fundamental a la hora de explicar cómo las personas interpretan y actúan en sus mundos sociales (Smith, et al., 2012). Las normas sociales se pueden llegar a convertir en guías internas de conducta y, en este sentido, vincularse con la obligación moral. Al interiorizase, adquieren un poder de influencia sobre las respuestas de los individuos que son independientes de presiones del entorno (véase Abrams y Levine, 2012; Mols, 2020).

En función de lo expuesto en este último epígrafe, el tercer objetivo que se plantea es explorar la posible relación entre la obligación moral de participar socialmente y la intención de iniciar emprendimiento social.

Una vez expuesta la fundamentación de los objetivos planteados, se describe a continuación la investigación llevada a cabo para darles respuesta.

\section{Metodología}

Un total de 261 estudiantes universitarios que cursaban los grados de trabajo social, criminología y sociología participaron en el estudio. Sus edades iban de los 18 a los 29 años, con una media de edad de $19,80(\mathrm{DT}=2,07)$. El 15,7\% eran varones y el $83,9 \%$ mujeres. 
Dichos participantes cumplimentaron un cuestionario en papel que permitía medir las siguientes variables:

- Intención de desarrollar diferentes formas de participación social. En este caso, se solicitaba que estimaran la probabilidad de desarrollar en un futuro cercano 7 formas de participación social con una escala de respuesta tipo Likert de 7 puntos, donde 1 significaba "nada probable" y 7 "muy probable": 1) ser miembro de una asociación con fines sociales, 2) voluntariado en una ONG, 3) activismo o militancia en algún movimiento social o causa concreta, 4) ser miembro de un partido político, 5) ser miembro de una agrupación política alternativa (por ejemplo, candidatura de unidad popular u organización política asamblearia), 6) participar libremente sin establecer compromisos con entidades sociales, involucrándome en las causas que considere más importantes en cada momento, y 7) trabajar en una cooperativa de iniciativa social generando ideas y poniéndolas en práctica para resolver problemas presentes en la sociedad. La última forma de participación social se utilizó para ejemplificar el emprendimiento social.

- Experiencia previa en participación social. A través de una única pregunta se pedía que indicasen si en el pasado habían desarrollado algún tipo de participación social vinculada a problemas sociales.

- Obligación moral. Se utilizaron 6 ítems de la Escala de Obligación Moral de Sabucedo, et al. (2018). Para responder se utilizó una escala de respuesta tipo Likert de 7 puntos $(1=$ nada de acuerdo; $7=$ muy de acuerdo). El Alfa de Crombach fue de 0,84.

El procedimiento de recogida de datos fue no probabilístico, tuvo un carácter incidental. La participación de los estudiantes fue voluntaria y firmaron previamente un compromiso informado, donde se describían los objetivos del estudio y se les informaba de que sus datos serían tratados con total confidencialidad, respetando su anonimato.

Con relación a los análisis llevados a cabo, para el primer objetivo planteado se hicieron análisis descriptivos no sólo de la intención de emprender socialmente, sino también de la intención de desarrollar las otras formas de participación social evaluadas. Para lograr el segundo objetivo, se realizaron análisis correlacionales entre las intenciones de desarrollar las diferentes formas de participación social (correlaciones de Pearson). Sumado a ello, se realizó un análisis de diferencias de medias para muestras independientes (T de Student) con el objetivo de profundizar en la relación existente entre emprendimiento social y otras formas de participación. Finalmente, para analizar la relación entre obligación moral y emprendimiento social, se llevaron a cabo análisis correlacionales y análisis de mediación múltiple, donde la variable predictora era la obligación moral, la variable predicha la intención de emprender socialmente, y las variables mediadoras las intenciones de desarrollar en el futuro las otras formas de participación social estudiadas. Para estos últimos análisis se utilizó el procedimiento descrito por Hayes (2013), concretamente el Modelo 4 de la macro PROCESS. Estos análisis asumen que la variable predictora influye a las variables mediadoras, y éstas a su vez lo hacen con la variable predicha, en este caso la intención de emprender socialmente (Preacher y Hayes, 2008).

\section{Resultados}

En la Tabla 1 se encuentran recogidos los estadísticos descriptivos y las correlaciones entre las diferentes intenciones de participar socialmente y la obligación moral experimentada con relación a ello. Las disposiciones a ser miembro de una asociación, hacer voluntariado y participar libremente son las más altas, aunque en general se consideran medias atendiendo a la escala de respuesta utilizada. Con relación a la disposición a emprender socialmente, esta sólo estaría por encima de ser miembro de un partido político o de una agrupación política alternativa. La variabilidad en todos los casos se considera similar y reducida, excepto en el caso de la participación política tradicional o alternativa. 
Tabla. 1. Estadísticos descriptivos y correlacionales.

\begin{tabular}{|c|c|c|c|c|c|c|c|c|}
\hline VARIABLES & 1 & 2 & 3 & 4 & 5 & 6 & 7 & 8 \\
\hline 1. Miembro asociación & -- & & & & & & & \\
\hline 2. Voluntario &, $48^{* *}$ & -- & & & & & & \\
\hline 3. Activista &, $40 * *$ &, $24 * *$ & -- & & & & & \\
\hline $\begin{array}{l}\text { 4. Miembro partido } \\
\text { político. }\end{array}$ & ,03 &,$- 14^{*}$ &, $20 * *$ & -- & & & & \\
\hline $\begin{array}{l}\text { 5. Miembro agrupación } \\
\text { política alternativa. }\end{array}$ &, $15^{*}$ &,$- 17 * *$ &, $35 * *$ &, $77 * *$ & -- & & & \\
\hline $\begin{array}{l}\text { 6. Participación social } \\
\text { libre. }\end{array}$ &, $34^{* *}$ &, $18^{* *}$ &, $40^{* *}$ &, $14^{*}$ &, $19 * *$ & -- & & \\
\hline 7. Emprendedor social. &, $43^{* *}$ &, $30 * *$ &, $41 * *$ &, $19 * *$ &, $24 * *$ &, $43^{* *}$ & -- & \\
\hline 8. Obligación moral. &, $35^{* *}$ &, $22 * *$ &, $35 * *$ &,- 02 &, 10 &, $37 * *$ &, $23 * *$ & -- \\
\hline M & 4,16 & 4,01 & 3,80 & 1,81 & 1,74 & 4,26 & 3,53 & 4,26 \\
\hline DT & 1,76 & 1,88 & 1,76 & 1,41 & 1,36 & 1,75 & 1,66 & 1,28 \\
\hline
\end{tabular}

Nota. $* \mathrm{p}<.05$.

Fuente: Elaboración propia.

Respecto a la relación existente entre las diferentes disposiciones a hacer participación social, se puede comprobar (ver Tabla 1) que la intención de emprender socialmente correlaciona significativamente y de forma positiva con todos los tipos de participación social. En mayor medida lo hace con ser miembro de una asociación y participar libremente, y en menor medida con las formas de participación política.

El análisis de diferencias de medias muestra que hay diferencias significativas en la disposición a emprender socialmente entre aquellos que tienen experiencias previas $(\mathrm{M}=3,86 ; \mathrm{DT}=1,71)$ en participación social y aquellos que no las tienen $(\mathrm{M}=3,27$; $\mathrm{DT}=1,57)(\mathrm{t}(260)=-2,919 ; \mathrm{p}<, 01)$. Los primeros (el 43,7\% de los participantes) son los que tienen una mayor disposición a emprender socialmente. Este resultado refuerza la relación ya mostrada previamente respecto a las correlaciones entre emprendimiento social y otras formas de participación social.

En cuanto a la relación que mantiene el emprendimiento social con la obligación moral para participar socialmente, la Tabla 1 muestra una correlación significativa y positiva, pero de menor intensidad si se compara con la hallada en otras formas de participación social, como son ser miembro de una asociación, ser activista o participar libremente.

La Tabla 2 sintetiza los resultados del análisis de mediación y aporta más información sobre la relación existente entre las variables objeto de análisis. En este sentido, se comprueba que la obligación moral no predice de forma significativa y directa el emprendimiento social, pero sí lo hace a través de su relación con otras formas de participación social estudiadas, excepto en los casos de ser miembro de un partido político o de una agrupación política alternativa. El efecto de la obligación moral sobre el emprendimiento social está mediado por la intención de desarrollar otras formas de participación social más tradicionales. 
Tabla. 2. Resumen de análisis de mediación para identificar los efectos indirectos de la obligación moral sobre la intención de emprender socialmente.

\begin{tabular}{clcc}
\hline \multicolumn{1}{c}{ Predictor } & \multicolumn{1}{c}{ Mediadores } & Consecuencia & Effect $[95 \%$ CIs] \\
\hline Obligación moral & ---- & Emprendimiento social &,$- 06[-, 21 ;, 08]$ \\
Obligación moral & Total & Emprendimiento social &, $36[, 24 ;, 47]$ \\
& Miembro de asociación & &, $08[, 01 ;, 16]$ \\
& Voluntario &, $04[, 00 ;, 10]$ \\
& Activista &, $08[, 02 ;, 17]$ \\
& Miembro de partido político &, $00[-, 02 ; 01]$ \\
& Miembro de agrupación &, $01[-, 01 ; .04]$ \\
& política alternativa &, $13[, 06 ;, 21]$ \\
\hline
\end{tabular}

Fuente: Elaboración propia.

\section{Discusión}

La presente investigación plantea el análisis de un tema que ha sido escasamente abordado en el estudio del emprendimiento social, la relación entre emprendimiento social y otras formas de participación social. Los resultados hallados muestran que el emprendimiento social mantiene una relación significativa y positiva con todas las formas de participación social estudiadas. Por ejemplo, la intención de ser miembro de una asociación, voluntario o activista se asociaría con la intención de emprender socialmente en el futuro. Sumado a ello, también se encuentra una relación entre la experiencia previa de participación social y la intención de emprender socialmente. Estos resultados ponen de manifiesto las conexiones que existen entre diferentes formas de participación social en la línea de lo descrito por otros autores (Haugh y Talwar, 2016; Makua et al., 2017, por ejemplo). En todos los casos, su objetivo es generar un cambio o promover una transformación social, lo que cambia es el "vehículo" que se utiliza para llegar al destino.

Con relación al análisis del calado que la cultura del emprendimiento pueda tener entre los jóvenes españoles, los datos muestran que la intención de emprender socialmente es media y está por debajo de otras formas de participación social cívica, como el voluntariado y el asociacionismo, pero por encima de otras con una orientación más sociopolítica como son ser miembro de un partido político o de una agrupación política alternativa. Estos resultados muestran una mayor propensión en los jóvenes a iniciar actividades de carácter cívico que sociopolítico, en la misma línea que lo hallado por otros estudios (Henn y Foard, 2014; Pavlova y Silbereisen, 2015). Pavlova y Silbereisen (2015) describen que, mientras que la participación cívica es percibida como una práctica desinteresada, moralmente pura y fácil de desarrollar, la participación política se ve como más difícil e incluso se la llega a considerar como un "negocio sucio" con métodos poco claros y cuestionables moralmente. En este sentido, aunque la disposición a emprender fuese menor en comparación a otras formas de participación cívica, quizás podría inferirse que su imagen no es tan negativa como la que tienen las fórmulas de participación señaladas.

Si la obligación moral se concibe como una motivación que no sólo deriva de ciertos valores personales, sino de la interiorización o cristalización de experiencias sociales (Zlobina y Sánchez, 2020) como la percepción de normas sociales, por ejemplo, podría interpretarse que la cultura del emprendimiento social todavía no tiene un hondo calado entre los jóvenes españoles, ya que la obligación moral no predice de forma directa la intención de emprender socialmente. Su impacto en el emprendimiento social viene mediado por su relación directa con la disposición a desarrollar otros tipos de participación social.

\section{Conclusiones}

Los resultados hallados en la presente investigación son coherentes con el hecho de que el emprendimiento social es una fórmula de intervención social relativamente novedosa en España. Pero lo esperable es que, siguiendo la estela de otros países anglosajones, el emprendimiento social se siga desarrollado en nuestro país. El documento de conclusiones del Consejo de la Unión Europea "La promoción de la economía social como motor clave del desarrollo económico y social en Europa" incide en la necesidad de impulsar este 
modelo de economía en la agenda política de los países miembros (Díaz y Lejarriaga, 2018). Como se ha descrito previamente, en el emprendimiento social se puede aunar el interés por combatir los problemas sociales, con el interés de generar un nicho de empleo sobre todo en los más jóvenes, contribuyendo de esta manera también a combatir el problema social concreto del desempleo juvenil. El emprendimiento social podría promover dinámicas que favorezcan la inserción sociolaboral de los jóvenes y, a un mismo tiempo, estimular formas alternativas de hacer economía, de entender la productividad, de fomentar la cooperación y de concebir el bien común.

Ya ha habido iniciativas previas que han mostrado la utilidad de otras formas de participación social, como es el caso del voluntariado, para promover la inserción laboral. Por ejemplo, la Plataforma del Voluntariado de España cuenta con la iniciativa Vol+, que permite evaluar las competencias que las personas han adquirido al hacer voluntariado. De esta forma, visibiliza que el voluntariado promueve la adquisición de competencias profesionales, lo que mejora la empleabilidad de las personas voluntarias. Holdsworth y Breweis (2014) y Pavlova y Silbereisen (2014) destacan la necesidad de considerar las funciones que puede tener la práctica del voluntariado con relación al trabajo remunerado.

Sobre la base de los resultados hallados, si el objetivo es promover el emprendimiento social entre los jóvenes, sería muy útil promover entre ellos el desarrollo de participación social más tradicional. Morales y Montes (2013) describen que los jóvenes tienden a ver el emprendimiento social como una postura frente a la vida. Quizás el objetivo sea precisamente ayudar a construir esa postura frente a la vida. Smith et al. (2016) describen que la decisión de emprender socialmente es con frecuencia motivada por percepciones morales y éticas de las personas, y en este sentido algunas investigaciones se han centrado en el rol de la compasión como un antecedente motivacional del emprendimiento social. En esta línea, Hockerts (2017) describe que la experiencia previa con problemas sociales es un predictor de la intención de emprender socialmente. Tales experiencias generan familiaridad con el tipo de problemas que las empresas sociales pretenden resolver, y, a su vez, hacen más probable la formación de la intención de resolver estos problemas. De esta forma, las intervenciones que van dirigidas a proporcionar a las personas un contacto directo con los problemas sociales contribuirán a promover el emprendimiento social (Hockerst, 2017).

Sumado a ello, si se consideran los posibles riesgos asociados a la extensión de una cultura exclusivamente economicista del emprendimiento a los que se ha aludido previamente, sería importante y deseable promover entre los jóvenes otros modos de "hacer emprendimiento" (Bruni, Gherardi y Poggio, 2005) vinculados a la participación cívica. El emprendimiento social podría servir para la reformulación de una acepción individualizadora y despolitizadora del emprendimiento, podría cuestionar la aspiración a hacer de la racionalidad productivista de la cultura emprendedora una ética referencial universal, podría reformular las asimétricas relaciones entre diversos grupos sociales (Lozano, Raufflet y González, 2018) y, finalmente, podría problematizar una única forma de entender qué significa desarrollo, el bien común, crecimiento, bienestar (Sanchis y Campos, 2018; Zamagni, 2018). Por ello, la eficacia emancipatoria de estas iniciativas depende tanto de su capacidad transformadora como de su vínculo con una dimensión ética y de justicia social. No obstante, a pesar del potencial de estas experiencias, en ocasiones estas se insertan en racionalidades que les hacen rehenes de las lógicas que justamente intentan combatir (Bruni et al, 2005; Lozano et al, 2018). En algunos casos se ha cuestionado la dimensión transformadora de algunas iniciativas de emprendimiento social, particularmente aquellas que se inscriben en un enfoque de economía capitalista inclusiva (Portales, 2018).

El emprendimiento social se presenta por tanto como una nueva "fórmula" que no sólo pasa a formar parte del entramado de formas de participación social que tienen como objetivo afrontar determinados problemas sociales, sino que también en muchos casos se funde con el activismo o los movimientos sociales, por ejemplo, y se convierte en un ingrediente esencial para la supervivencia de las entidades de acción social. Este trabajo evidencia el interés de abordar la relación entre emprendimiento social con otros tipos de participación social, pero aún queda mucho recorrido por llevar a cabo en este sentido y las propuestas de futuros estudios en esta línea pueden seguir caminos muy diferentes. Por ejemplo, podría ser interesante analizar las experiencias previas de participación social de las personas que ya han emprendido socialmente y considerar la perspectiva de género. Mientras que las diferencias entre varones y mujeres han sido ampliamente discutidas con relación al emprendimiento comercial (ver por ejemplo, García, Villaseca y González, 2019; Marlow, 2020), apenas han sido analizadas con relación al emprendimiento social (Lewis y Henry, 2019). Dos excepciones son el estudio de Fernández, López y San Martín (2020) y la revisión de Lewis y Henry (2019). En el primero, se describe que las mujeres emprendedoras tienden a enfatizar la creación de valor social y a restar importancia a la creación de valor económico en mayor medida que los varones, y que esto, en función del contexto socioeconómico de cada país, podría dar lugar a una mayor o menor propensión a emprender socialmente en las mujeres. En el segundo, se defiende la necesidad de explicaciones multidimensionales a la hora de analizar la relación entre género y emprendimiento social que pongan el énfasis tanto en dimensiones de nivel micro como macro (cuestiones actitudinales como el rechazo de estructuras jerárquicas androcéntricas y la extensión de sus roles sociales; pero también influencias estructurales como el contexto geográfico de procedencia). Si atendemos a las diferencias en función del 
género que se encuentran en otras formas de participación social (ver por ejemplo, Dávila, Zlobina y Álvarez, 2020), el interés por abordar la perspectiva de género en esta temática se incrementa. También podría ser relevante abordar otras cuestiones apuntadas previamente, como el análisis de en qué medida y a través de qué procesos el emprendimiento social promueve realmente el cambio social (Haugh y Talwar, 2016), o abordar el emprendimiento social colaborativo o colectivo en contraposición al individual (Montgomery et al. 2012; ver en Pless, 2012).

La presente investigación cuenta con una serie de limitaciones. Se ha utilizado una estimación de la probabilidad de iniciar diferentes tipos de participación social y no una medida de la participación real en esos casos. Pero, tal y como afirman las teorías de la Acción Razonada y la Acción Planificada, la predicción de una conducta está mediada por la intención de realizar esa conducta. Sumado a ello, dada la complejidad, amplitud e indefinición de lo que se puede entender como emprendimiento social, quizás la formulación del ítem que alude a la intención de emprender socialmente podía haberse precisado de otra manera, aunque se utilizó una de las formas de emprendimiento social más frecuentes actualmente en nuestro país. También su planteamiento pudo mostrar el emprendimiento social como una forma más de participación social, que como una alternativa de empleo. Otra cuestión a tener en cuenta es el perfil de los jóvenes que participaron en el estudio, todos ellos universitarios. El nivel educativo de los jóvenes puede haber afectado a su mayor o menor disposición a emprender socialmente y a desarrollar otras formas de participación social. Por ejemplo, el nivel educativo se asocia estrechamente con la práctica de voluntariado (Dávila y Chacón, 2003) y se ha encontrado que los emprendedores sociales tienden a tener altos niveles educativos (Bosma, et al., 2016). Igualmente, la mayoría de los participantes eran mujeres, pero la investigación previa muestra que la brecha de género en el emprendimiento social es significativamente menor que en la actividad empresarial comercial (Bosma, et al., 2016), aunque los tipos de emprendimiento social (dimensión del emprendimiento a pequeña o gran escala-, modalidades -procomunitarios o innovación social-, y formato -en organizaciones de base a nivel local o a escala global-) varían en gran medida en función del género (Lewis y Henry, 2019).

La investigación que aquí se presenta es, en gran medida, descriptiva y muestra una imagen limitada de un fenómeno en ciernes en nuestro país, pero creemos que muestra la relevancia de un nuevo foco de interés a considerar por todos aquellos profesionales o académicos vinculados y/o interesados en el tercer sector. El emprendimiento social es un ejemplo más de una realidad cambiante que se adapta y se transforma para dar respuesta a nuevas demandas sociales.

\section{Referencias bibliográficas}

Abrams, D. y Levine, J. M. (2012) Norm formation: Revisiting Sherif's autokinetic illusion study. En: Smith, J.R.; Haslam, S.A. (Eds.), Psychology: Revisiting the classic studies. Social psychology: Revisiting the classic studies. New York: Sage Publications Ltd, pp. 57-75.

Alvord, S., Brown, L.D. y Letts, C.W. (2004) Social Entrepreneurship and Societal Transformation: An Exploratory Study. The Journal of Applied Behavioral Science, vol. 40, $\mathrm{N}^{\circ}$ 3, pp. 260-282. https://doi.org/10.1177/0021886304266847.

Andersson, F.O. y Self, W. (2015) The Social-Entrepreneurship Advantage: An Experimental Study of Social Entrepreneurship and Perceptions of Nonprofit Effectiveness. Voluntas, vol. 26, pp. 2718-2732. https://doi.org/10.1007/s11266-014-9543-1.

Austin, J.A., Stevenson, H. y Wei-Skillern, J. (2006) Social and Commercial Entrepreneurship: Same, Different, or Both? Entrepreneurship: Theory and Pratice Journal, vol. 30, No 1, pp. 1-22. https://doi.org/10.1111/j.15406520.2006.00107.x.

Boeri, N. (2018) Challenging the gendered entrepreneurial subject. Gender, Development and the Informal Economy in India. Gender and Society, vol. 32, № 2, pp. 157-179. https://doi.org/10.1177/0891243217750119.

Bosma, N., Schøtt, T., Terjesen, S. y Kew, P. (2016) Special topic report social enterpreneurship. https://www.gemconsortium.org/file/open?fileId=49542. Fecha de consulta: 12/10/2020.

Briales, A. (2017) Emprendeudores fracasados: individualización neoliberal en los discursos sobre el desempleo. RECERCA. Revista de Pensament i Anàlisi, vol. 20, pp. 79-104. https://doi.org/10.6035/Recerca.2017.20.5.

Bruni, A., Gherardi, S. y Poggio, B. (2005) Doing Gender, Doing Entrepreneurship: An Ethnographic Account of Intertwined Practices. Gender, Work and Organization, vol. 11, $\mathrm{N}^{\mathrm{o}}$ 4, pp. 407-429. http://citeseerx.ist.psu.edu/viewdoc/download?doi=10.1.1.864.6493\&rep=rep1\&type=pdf.

Cabasés, M.A., Pardell, A. y Feixa, C. (eds.) (2018) Jóvenes, trabajo y futuro. Perspectivas sobre la garantía juvenil en España y Europa. Valencia: Tirant lo Blanch.

Carrasco, I., y Buendía, I. (2020) Political Activism as Driver of Cooperative Sector Inmaculada Carrasco. Voluntas, vol. 31, pp. 601-613. https://doi.org/10.1007/s11266-020-00198-6.

Dávila, M.C. y Chacón, F. (2003) Diferencias entre voluntarios ambientales y socioasistenciales en el perfil sociodemográfico. Revista de Psicología Social Aplicada, vol. 13, No 3, pp. 19-44. 
Dávila, M.C., Zlobina, A., y Álvarez, G. (2020) La influencia diferencial de las redes sociales en la participación social de mujeres y varones. Redes: Revista Hispana para el Análisis de Redes Sociales, vol. 31, No 1, pp. 1-18. https://doi.org/10.5565/rev/redes.835.

Dees, J.G. (2012) A Tale of Two Cultures: Charity, Problem Solving, and the Future of Social Entrepreneurship. Journal of Business Ethics, vol. 111, No 3, pp. 321-334. https://www.jstor.org/stable/23325671.

Díaz, F. y Lejarriaga, G. (2018) Emprendimiento social y empleabilidad. REVESCO. Revista de Estudios Cooperativos, No 129, pp. 9-15. http://dx.doi.org/10.5209/REVE.62962.

Elsayed, Y. (2018) At the Intersection of Social Entrepreneurship and Social Movements: The Case of Egypt and the Arab Spring Yomna Elsayed1. Voluntas, vol. 29, pp. 819-831. https://doi.org/10.1007/s11266-017-9943-0.

Escudero, R. (2016) El Sistema Nacional de Garantía Juvenil: entre un amplio número de medidas y unos resultados aún insuficientes. En: Escudero, R. Jóvenes y empleo. Una mirada desde el derecho, la sociología y la economía. Madrid: Centro Reina Sofía sobre Adolescencia y Juventud, pp.50-60.

Eurofound (2020) Living, working and COVID-19. Luxembourg: Publications Office of the European Union. https://www.eurofound.europa.eu/publications/report/2020/living-working-and-covid-19.

Eurofound (2015) Youth entrepreneurship in Europe: values, attitudes and policies. Luxemburgo: Publications Office of the European Union. https://www.eurofound.europa.eu/es/publications/report/2015/labour-market/youthentrepreneurship-in-europe-values-attitudes-policies.

Fernández, A., López, C. y San Martín, P. (2020) The Moderating Effect of Countries' Development on the Characterization of the Social Entrepreneur: An Empirical Analysis with GEM Data. Voluntas, vol. 31, № 3, pp. 563-580. DOI:10.1007/s11266-020-00216-7.

García, J.C., Villaseca, D. y González, S. (2019) Emprendimiento femenino y financiación social: un estudio comparado. REVESCO. Revista de Estudios Cooperativos, № 132, pp. 97-121. https://doi.org/10.5209/reve.65974.

Gawell, M. (2013) Social Entrepreneurship: Action Grounded in Needs, Opportunities and/or Perceived Necessities? Voluntas, vol. 24, pp. 1071-1090. DOI 10.1007/s11266-012-9301-1.

Gupta, P., Chauhanb, S., Paulc, J. y Jaiswald, M.P. (2020) Social entrepreneurship research: A review and future research agenda. Journal of Business Research, vol. 113, pp. 209-229. https://doi.org/10.1016/j.jbusres.2020.03.032.

Guzmán, A., y Trujillo, M. A. (2008) Emprendimiento social: Revisión de literatura. Estudios Gerenciales, vol. 24, N ${ }^{\circ}$ 109, pp.105-125. https://www.redalyc.org/pdf/212/21211518005.pdf.

Harris, P., Renko, M. y Caldwell, K. (2014) Social entrepreneurship as an employment pathway for people with disabilities: exploring political economic and socio-cultural factors. Disability \& Society, vol. 29, No 8, pp. 2751290. DOI:10.1080/09687599.2014.924904.

Haugh, H.M. y Talwar, A. (2016) Linking Social Entrepreneurship and Social Change: The Mediating Role of Empowerment. Journal of Business Ethics, vol. 133, pp. 643-658. DOI 10.1007/s10551-014-2449-4.

Hayes, A. F. (2013) Methodology in the social sciences. Introduction to mediation, moderation, and conditional process analysis: A regression-based approach. New York: Guilford Press.

Henar, L. (coord.) (2018) II Evaluación de la Iniciativa de Empleo Juvenil. Madrid: Ministerio de Trabajo, Migraciones y Seguridad Social y https://www.fresnoconsulting.es/upload/94/47/Informe_II_Evaluacixn_IEJ_2018.pdf.

Henn, M. y Foard, N. (2014) Social differentiation in young people's political participation: the impact of social and educational factors on youth political engagement in Britain. Journal of Youth Studies, vol. 17, No 3, pp. 360-380. https://doi.org/10.1080/13676261.2013.830704.

Hockerts, K. (2017) Determinants of Social Entrepreneurial Intentions. Entrepreneurship Theory and Practice, vol. 41, No1, pp. 105-130. https://doi.org/10.1111/etap.12171.

Holdsworth, C. y Brewis, G. (2014) Volunteering, choice and control: a case study of higher education student volunteering. Journal of Youth Studies, vol. 17, No 2, pp. 204-219. https://doi.org/10.1080/13676261.2013.815702.

Hota, P.K., Subramanian, B. y Narayanamurthy, G. (2020) Mapping the Intellectual Structure of Social Entrepreneurship Research: A Citation/Co-citation Analysis. Journal of Business Ethics, vol. 166, pp. 89-114. https://doi.org/10.1007/s10551-019-04129-4.

Kapusuz A. G., Çavuş M. F. y Pekkan N. Ü. (2018) Perceived social support and social entrepreneurship: Gender perspective from Turkey. En: Ratten, V.; Graga, V.; Marques, C.S. Knowledge, Learning and Innovation. New York: Springer International Publishing AG, pp. 75-90.

Ko, W. y Liu, G. (2020) The transformation from traditional nonprofit organizations to social enterprises: An institutional entrepreneurship perspective. Journal of Business Ethics, vol. 30. DOI:10.1007/s10551-020-04446-z.

Kraus, S., Filser, M., O’Dwyer, M. y Shaw, E. (2014) Social Entrepreneurship: An exploratory citation analysis. Review of Managerial Science, vol. 8, pp. 275-292. DOI 10.1007/s11846-013-0104-6.

Lewis, K.V. y Henry, C. (2019) Gender and social entrepreneurship research: contemporary themes. En de Bruin, A.; Teasdale, S. A research agenda for social entrepreneuship. Cheltenham: Edward Elgar Publishing Limited, pp. 121127.

Lozano, J. F., Raufflet, E., y González, E. (2018) Lecturas críticas de las nuevas propuestas de economía alternativa: ¿Qué hay de nuevo en lo nuevo? RECERCA. Revista De Pensament I Anàlisi, vol. 23, pp. 918. https://doi.org/10.6035/Recerca.2018.23.1.

Mair, J. y Marti, I. (2006) Social Entrepreneurship Research: A Source of Explanation, Prediction, and Delight. Journal of World Business, vol. 4, No 1, pp. 36-44. DOI: 10.1016/j.jwb.2005.09.002. 
Mair, J. y Marti, I. (2009) Entrepreneurship in and around institutional voids: A case study from Bangladesh. Journal of $\begin{array}{llllll}\text { Business } \quad \text { Venturing, vol. } & \text { N } & \text { N } & 5, & \text { pp. } & 419-435 .\end{array}$ https://managementdelaculture.files.wordpress.com/2016/11/entrepreneurship_in_and_around_institutional_voids_a case study from bangladesh.pdf.

Mair, J. y Noboa, E. (2003) Social Entrepreneurship: How Intentions to Create a Social Enterprise Get Formed. https://ssrn.com/abstract=462283 or http://dx.doi.org/10.2139/ssrn.462283. Fecha de consulta: 15/09/2020.

Mair, J., y Noboa, E. (2006) Social Entrepreneurship: How Intentions to Create a Social Venture Get Formed. En: Mair, J.; Robinson, J.; Hockerts, K. Social Entrepreneurship. New York: Palgrave MacMillan, pp. 121-136.

Makua, A., Cuenca-Amigo, M. y San Salvador del Valle, R. (2017) Relación entre las prácticas de ocio significativas de la juventud y el emprendimiento social. El caso Think Big Jóvenes. OBETS: Revista de Ciencias Sociales, vol. 12, pp. 151-176. DOI:10.14198/OBETS2017.12.1.16.

Marlow S. (2020) Gender and entrepreneurship: Past achievements and future possibilities. International Journal of Gender and Entrepreneurship, vol. 12, № 1, pp. 39-52. https://doi.org/10.1108/IJGE-05-2019-0090.

Martin, R. y Osberg, S. (2007) Social Entrepreneurship: The Case for Definition. Stanford Social Innovation Review, vol. 5, No 28. https://www.law.berkeley.edu/php-programs/courses/fileDL.php?fID=7288.

Martínez, L. (2017) El discurso europeo sobre el emprendimiento. Políticas públicas, trabajo y subjetividad en el marco de la gobernanza económica europea. Tesis doctoral. Universidad Pública de Navarra. https://academicae.unavarra.es/xmlui/handle/2454/28809.

McMillan, I. A. (2003) Social Entrepreneurs: Playing the Role of Change Agents in Society. https://knowledge.wharton.upenn.edu/article/social-entrepreneurs-playing-the-role-of-change-agents-in-society/. Fecha de consulta: 15/09/2020.

Mols, F. (2020). Behaviour Change. En: Jetten, J.; Reicher, S.D.; Haslam, S.A.; Cruwys, T. Together Apart: The Psychology of COVID-19. London, UK: Sage, pp. 36 - 40.

Montesano, N. (2016) A theoretical and methodological approach to social entrepreneurship as world-making and emancipation: social change as a projection in space and time. Entrepreneurship \& Regional Development, vol. 28, No 7-8, pp. 536-562. DOI: 10.1080/08985626.2016.1221229.

Morales, A., y Montes, J. (2013) Valores, actitudes y motivaciones en la juventud ante el emprendimiento individual y colectivo. REVESCO. Revista de Estudios Cooperativos, $\mathrm{N}^{\mathrm{o}} \quad 112, \quad \mathrm{pp} . \quad 11-35$. http://dx.doi.org/10.5209/rev_REVE.2013.v112.43062.

National Agency for European Educational Programmes and Mobility (2017). Social entrepreneurship as an alternative for Young unemployed.

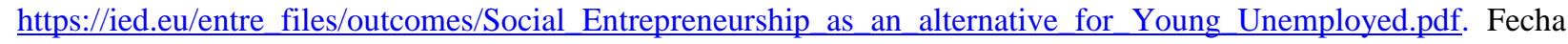
de consulta: 15/09/2020.

Nga, J.K.H. y Shamuganathan, G. (2010) The Influence of Personality Traits and Demographic Factors on Social Entrepreneurship Start Up Intentions. Journal of Business Ethics, vol. 95, pp. 259-282. DOI 10.1007/s10551-0090358-8.

Ortiz, P y Olaz, P. (2018) Factores competenciales en el emprendimiento de la mujer. Revista Española de Sociología, vol. 27, No 3, pp. 413-432. https://doi.org/10.22325/fes/res.2018.21.

Pareja, B. (2013) El emprendimiento social: una introducción a los conceptos, dimensiones y teorías. Servicios sociales y políticas social, vol. 102, pp. 11-22. https://www.serviciossocialesypoliticasocial.com/el-emprendimiento-socialuna-introduccion-a-los-conceptos-dimensiones-y-teorias.

Pavlova, M.K. y Silbereisen, R.K. (2014) Coping with occupational uncertainty and formal volunteering across the life span. Journal of Vocational Behavior, vol. 85, pp. 93-105. DOI: 10.1016/j.jvb.2014.05.005.

Pavlova, M.K. y Silbereisen, R.K. (2015) Supportive Social Contexts and Intentions for Civic and Political Participation: An Application of the Theory of Planned Behaviour. Journal of Community \& Applied Social Psychology, vol. 25, pp. 432-446 https://doi.org/10.1002/casp.2223.

Pless, N.M. (2012) Social Entrepreneurship in Theory and Practice-An Introduction. Journal of Business Ethics, vol. 111, pp. 317-320. DOI 10.1007/s10551-012-1533-x.

Portales, L. (2018). Emprendimiento social, ¿alternativa o continuidad del sistema neoliberal? RECERCA. Revista de Pensament $i$ Anàlisi, No 23, pp. 43-66. http://dx.doi.org/10.6035/Recerca.2018.23.3.

Portuguez, M., Valenzuela, J.R. y Navarro, C. (2018) Diseño y validación de un test de evaluación del potencial en emprendimiento social. REVESCO. Revista de Estudios Cooperativos, $\mathrm{N}^{\mathrm{o}}$ 128, pp. 192-211. DOI: 10.5209/REVE.60207.

Preacher, K.J. y Hayes, F.H. (2008) Asymptotic and resampling strategies for assessing and comparing indirect effects in multiple mediator models. Behavior Research Methods, vol. 40, No 3, pp. 879-891. DOI: 10.3758/BRM.40.3.87.

Robin Hood Stores: cuando hacer la compra es activismo anticapitalista (2020, 28 de Noviembre). El Diario.es. https://www.eldiario.es/economia/compra-activismo-anticapitalista_1_6466375.html. Fecha de consulta: $02 / 12 / 2020$.

Rodríguez, C. y Ramos, J. (2016) El sistema español de Garantía Juvenil y Formación Profesional Dual en el contexto de la Estrategia Europea de Empleo. Working Paper, ICEI, vol. 05/15.

Rodríguez, C., Serrano, A. y Martínez, L. (2020) Las éticas de la empreabilidad en el programa de Garantía juvenil en España: una perspectiva discursiva. Papers, pp. 1-18. http://dx.doi.org/10.5565/rev/papers.2797. 
Rodríguez, R.y Borges, E. (2018) El perfil del emprendedor. Construcción cultural de la subjetividad laboral postfordista. Cuadernos de Relaciones Laborales, vol. 36, № 2, pp 65-284. https://doi.org/10.5209/CRLA.60697.

Rodríguez, J. y Verd, J.M. (2018) El diseño y despliegue del sistema de garantía juvenil en España. Un análisis documental. Revista Española de Sociología, vol. 27, №3, pp. 395-412. https://doi.org/10.22325/fes/res.2018.22.

Romero, A.M. y Milone, M. (2016) El emprendimiento en España: Intención emprendedora, motivaciones y obstáculos. Revista de Globalización, Competitividad y Gobernabilidad, vol. $10, \quad \mathrm{~N}^{\mathrm{o}} \quad 1, \quad \mathrm{pp} . \quad 95-109$. https://www.redalyc.org/pdf/5118/511854473005.pdf.

Sabucedo, J. M., Dono, M., Alzate, M., y Seoane, G. (2018) The importance of protesters' morals: moral obligation as a key variable to understand collective action. Frontiers in Psychology, vol. 9, pp. 418. https://doi.org/10.3389/fpsyg.2018.00418.

Sánchez, J., Martín, S., Bel, P. y Lejarriaga, G. (2018) Educación y formación en emprendimiento social: características y creación de valor social sostenible en proyectos de emprendimiento social. REVESCO. Revista de Estudios Cooperativos, No 129, pp. 16-38. https://doi.org/10.5209/REVE.62492.

Sanchis, J. y Campos, C. (2018) Fonamentació teòrica del model de l'Economia del Bé Comú des de la perspectiva organitzativa. RECERCA. Revista de Pensament I Anàlisi, vol. 23, pp. 131-50. https://doi.org/10.6035/Recerca.2018.23.6.

Santamaría, E, y Carbajo, D. (2019) Emergencias de la crisis: figuras antiheroicas del emprendimiento juvenil en España. Política y Sociedad, vol. 56, No 1, pp. 191-211. https://doi.org/10.5209/poso.60030.

Santos, F.M. (2012) A Positive Theory of Social Entrepreneurship. Journal of Business Ethics, vol. 111, pp. 335-351. DOI 10.1007/s10551-012-1413-4.

Santos, A. (2014) La política en manos de los empresarios. El inseparable ascenso de la ideología del emprendedor. Papeles de Relaciones Ecosociales y Cambio Global, vol. 29, $\mathrm{N}^{\mathrm{o}} \quad 127, \quad \mathrm{pp} . \quad 29-43$. https://educaciotransformadora.files.wordpress.com/2016/04/la_politica_en_manos_de_los_empresarios.pdf.

Seelos, C. y Mair, J. (2004). Social entrepreneurship: The contribution of individual entrepreneurs to sustainable development. IESE Business School Working, No 553. DOI: 10.2139/ssrn.701181.

Seelos, C. y Mair, J. (2005) Social Entrepreneurship: Creating New Business Models to Serve the Poor. Business Horizons, № 48, Vol. 3, pp. 241-246. DOI: 10.1016/j.bushor.2004.11.006.

Santamaría, E. y Carbajo, D. (2019). Figuras antiheroicas del emprendimiento juvenil en España. Política y Sociedad, vol. 56, N N $^{\mathrm{o}}$, 191-211. https://doi.org/10.5209/poso.60030.

Serrano, A. y Martín-Martín, P. (2017) From employability to entrepreneurability. Youth in the spotlight in times of crisis Journal of Youth Studies, vol. 1, No 4, pp. 798-821. https://doi.org/10.1080/13676261.2016.1273513.

Serrano, A. y Fernández, C. (2018) De la metáfora del mercado a la sinécdoque del emprendedor. Cuadernos de Relaciones Laborales, vol. 36, № 2, pp. 207-224. https://doi.org/10.5209/CRLA.60694.

Smith, J.R. et al. (2012) Congruent or conflicted? The impact of injunctive and descriptive norms on environmental intentions. Journal of Environmental Psychology, vol. 32, № 4, pp. 353-361. DOI:10.1016/j.jenvp.2012.06.001.

Smith, B.R., Kistruck, G.M. y Cannatelli, B. (2016) The Impact of Moral Intensity and Desire for Control on Scaling Decisions in Social Entrepreneurship. Journal of Business Ethics, vol. 133, pp. 677-689. DOI 10.1007/s10551-0142447-6.

Stecker, M. J. (2014) Revolutionizing the nonprofit sector through social entrepreneurship. Journal of Economic Issues, vol. 48, No 2, pp. 349-358. https://doi.org/10.2753/JEI0021-3624480208.

Suárez, B. (2017) Autoempleo (y emprendimiento) juvenil: ¿ahuyentar a los jóvenes de los derechos y garantías laborales?. Cuadernos de Relaciones Laborales, vol. 35, № 1, pp. 151-164. https://doi.org/10.5209/crla.54987.

Tan, W.L., Williams, W. y Tan, T.M. (2005) Defining the 'Social' in 'Social Entrepreneurship': Altruism and Entrepreneurship. International Entrepreneurship and Management Journal, vol. 1, pp. 353-365. https://core.ac.uk/download/pdf/13242733.pdf.

Úbeda, M. y Sánchez, J. (2018) Representación y producción de subjetividades jóvenes: gramáticas de la empleabilidad y el emprendimiento en la Garantía Juvenil. En: Cabasés, M.A.; Pardell, A.; Feixa, C. Jóvenes, trabajo y futuro. Perspectivas sobre la garantía juvenil en España y Europa. Valencia: Tirant lo Blanch, pp. 53-75.

Valenzuela-García, H., Molina, J.L., Lubbers, M., Escribano, P. y Fuentes, S. (2019) Emprendimiento Social. Autoempleo y extracción del valor en la era post-crisis. Revista de Antropología Social, vol. 28, No 2, pp. 371-390. https://dx.doi.org/10.5209/raso.65619.

Velasco, L.C., Estrada, L.I., Pabón, M. y Tójar, J.C. (2019) Evaluar y promover las competencias para el emprendimiento social en las asignaturas universitarias. REVESCO. Revista de Estudios Cooperativos, No 131 , pp. 199-223. https://doi.org/10.5209/REVE.63561.

Wang, Q. y Aaltio, L. (2017) Social Entrepreneurship - Discourses and Contributions: A Literature Analysis. EJBO Electronic Journal of Business Ethics and Organization Studies, vol. 22, $\mathrm{N}^{\mathrm{o}} \quad 2, \quad \mathrm{pp} . \quad 14-23$. http://ejbo.jyu.fi/pdf/ejbo_vol22_no2_pages_14-23.pdf.

Weerawardena, J. y Mort, G.S. (2006) Investigating social entrepreneurship: A multidimensional model. Journal of World Business, vol. 41, pp. 21-35. DOI:10.1016/J.JWB.2005.09.001.

Zamagni, S. (2018). Civil Economy. A New Approach to the Market in the Age of the Fourth Industrial Revolution. RECERCA. Revista De Pensament I Anàlisi, vol. 23, pp. 151-168.

Zlobina, A. y Sánchez, S. (2020) Participación social feminista y ecologista: el papel de distintas normas sociales y de la obligación moral. IV Congreso Internacional de SCEPS, Burgos. 